Assiut University web-site: www.aun.edu.eg

\title{
DETECTION OF BLOOD PROTOZOA IN PET DOGS IN NINEVEH PROVINCE
}

\author{
WASAN AMJAD ALOBAIDII; RAFAL LUQMAN ALLHAIBI and \\ MARYAM AHMED ALKHERO \\ College of Veterinary Medicine, University of Mosul, Iraq
}

Received: 15 April 2019; Accepted: 27 Jun 2019

\begin{abstract}
Through out this study we collected 54 blood samples from the cephalic vein of dogs. They were all obtained from indoor breeding from both sexes and were of different ages and different breeds. Blood smears were prepared, stained with Giemsa stain and examined under the light microscope to detect blood parasites. The result of clinical examination of dogs showed different clinical signs which included nervous signs, weakness, haemglobinurea and Enlargement of lymph nodes, whereas others did not show any clinical signs. The clinical signs combined with presence of blood protozoa which included Babesia gibsoni, B.canis, Trypanosoma evansi, Hepatozoon canis, the high infection rate was recorded in Babesia (31.4\%); while the lowest infection rate was recorded in Trypanosoma evansi (11.2\%) Our results detected Hepatozoon canis in dogs (20.3\%), Some cases showed mixed infection with Trypanosoma evansi and Hepatozoon canis. A higher infection rate was found in older age with a significance of variance $p \leq 0.05$. Males showed higher infection rate than their female counterparts $(\mathrm{p} \leq 0.05)$ and large breeds showed higher infection rate than small breeds. We conclude that the pet dogs recorded high infective rate with blood protozoa and recommended the owner to use antiprotozoal drugs and ectoparasites spots and test the animal periodically in veterinary clinic to identify the infection early.

In conclusion, a high rate of infection with blood parasites was found in pet dogs. Vegular testing and routine treatment program of with antiprotozoal drugs and anti-parasitic drugs should be used a control measure.
\end{abstract}

Key words: Blood protozoa, dogs, antiprotozoal drugs

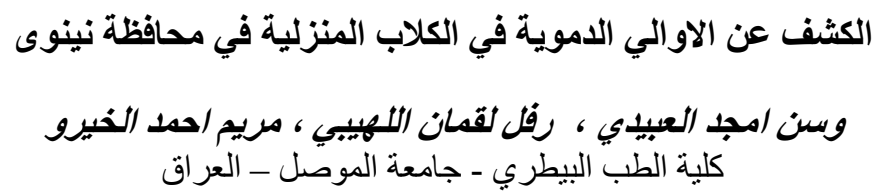

ABSTRACT

الخلاصة

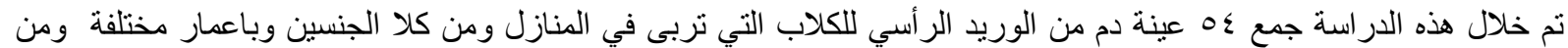

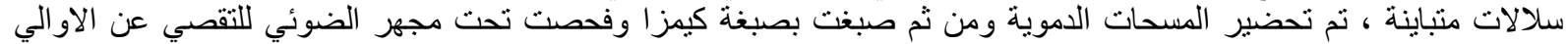

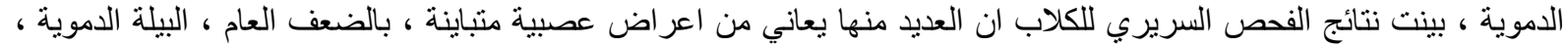

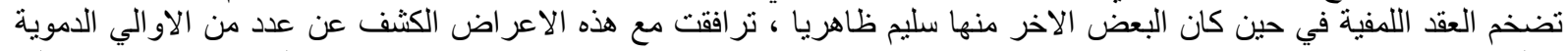

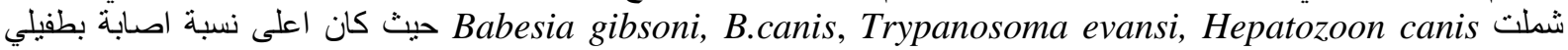
Babesia 31.4\% Hepatozoon canis

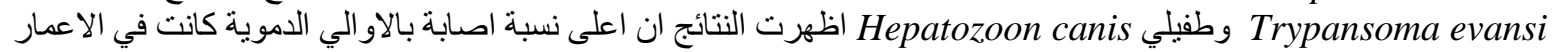




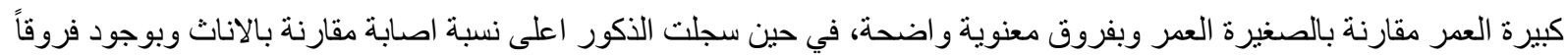

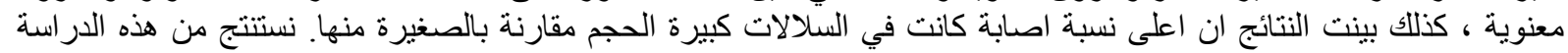

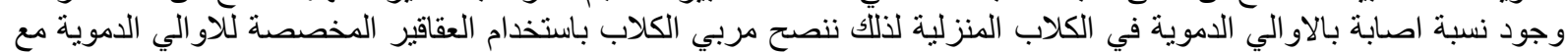

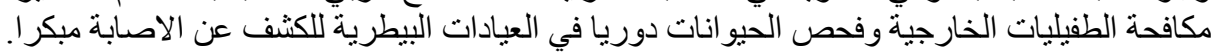

\section{INTRODUCTION}

\section{المقدمة}

تعد الكلاب احد الحيوانات الذكية والمصاحبة في تربيتها مع الانسان واستخدمت على مر العصور في حماية الانسان وحيواناته

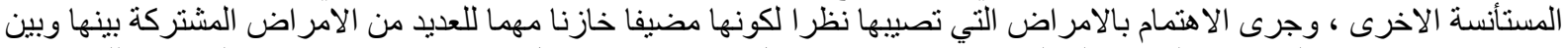

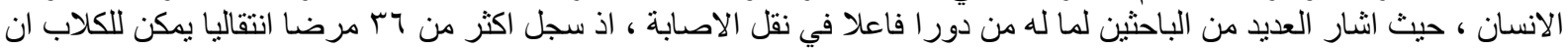

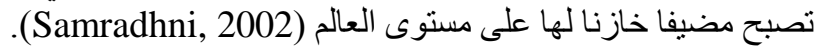

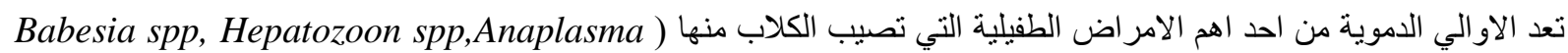

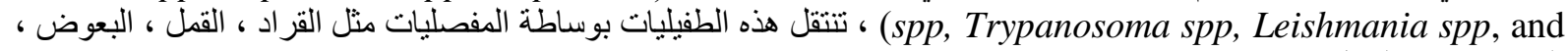
الذباب وذباب الرمل (Lan et al., 2017, Michal et al., 2010)

تسبب هذه الاو الي الاموية اعر اضا سريرية عديدة منها فقر الدم ، الهزال ، اعراضا عصبية ، اسهال ، تقيؤ ، تضخم العقد اللمفية ، اليرقان وفي بعض الاحيان تسبب الموت (Mahmud et al., 2014).

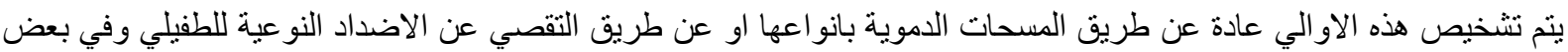

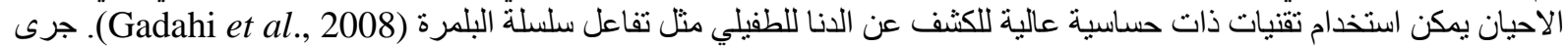

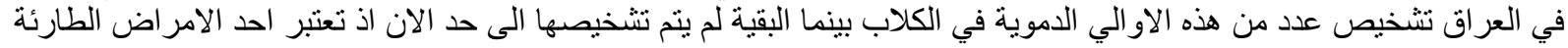

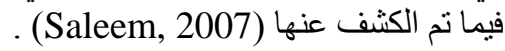

لوحظ خلال العامين الدنصرمين الاكثار من تربية الكلاب في المنازل في مدينة الموصل ومع هذه الزيادة في تربية الكلاب كانت

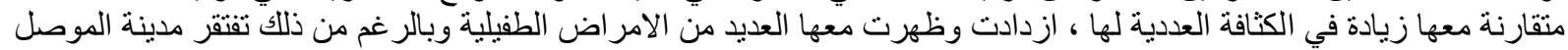

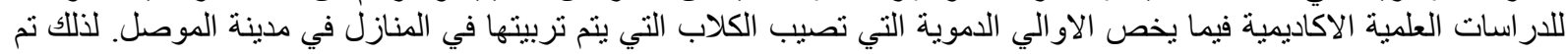

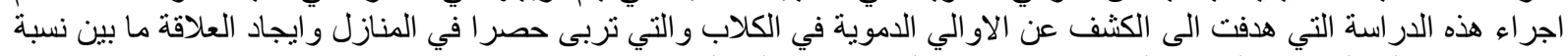
الاصابة بالاو الي الدموية والعمر ، الجنس ، الاعر اض الفئ السريرية ، و السلالة.

\section{MATERIALS AND METHODS المواد وطرائق العمل}

حيوانتات الدراسة الارسة

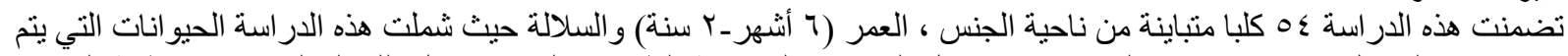

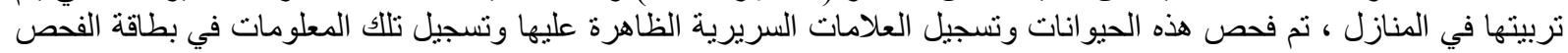

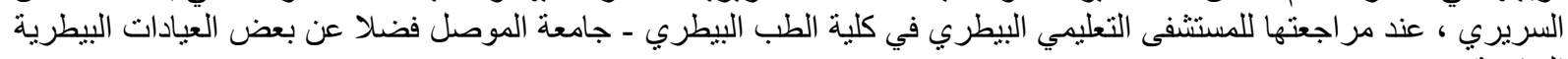
الخاصةة . الخرة

\section{جمع العينات} تم جمع الدم بواقع ه مل من الوريد الراسي Cephalic vein وضع الدم في انابيب تحوي على مانع تخثر EDTA وذلك لغرض تحضير المسحات الدموية.

تحضير المسحات الدموية الذفيفة وصبغها

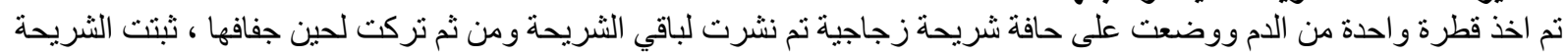
بعد ذللك باستخدام الكحول المثيلي المطلق لمدة ه دقائق، فضلا عن عمل مسحات دموية رطبة للكثف عن حركة عركة الطفيلي

.(Coles, 1986)

تمضير الخلايا اللمفية

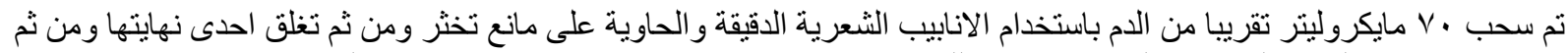

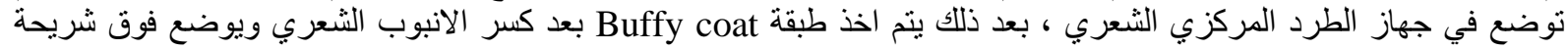

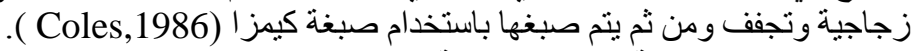

صبغ المسحات الاموية باستخدام صبغة كيمزا Giemsa stain 


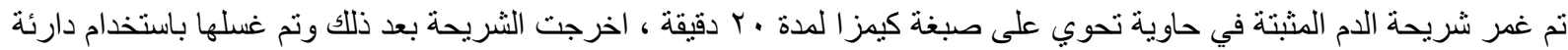
الفوسفات المتعادلة ، جفقت بعد ذلك وتم فحصها باستخدام المجهر الضوئي تحت قوى تكبير مختلفة وذلك للكثف عن اللفي الاو الي الدموية

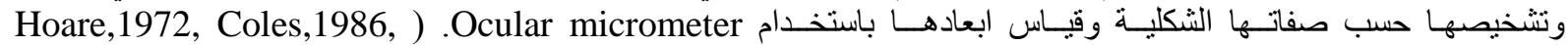

(Durate et al., 2016

التحليل الاحصائي

\section{RESULTS}

النتائج

نتائج الفحص السريري

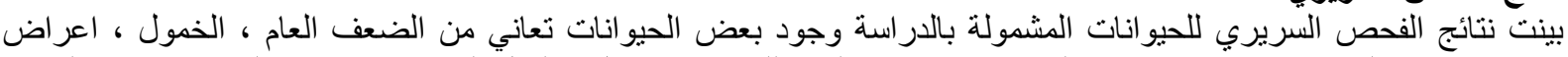

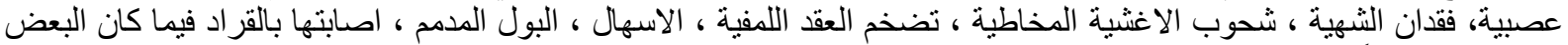

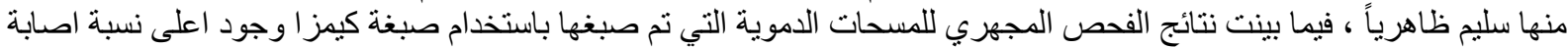

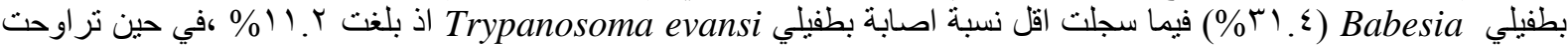

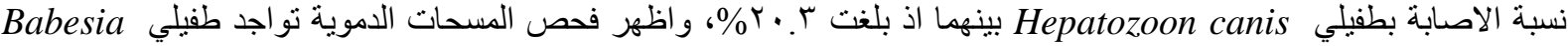

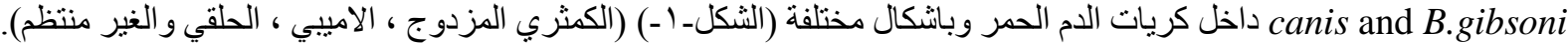

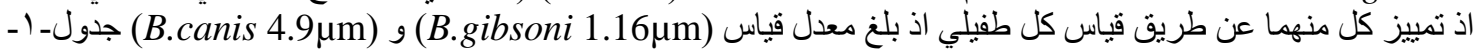

كما لوحظ طفيلي Trypanosoma evansi بشكله النموذجي الاسطو اني، اذ تم تمييزه حسب صفاته الثكلية والقياسية اذ كان معدل

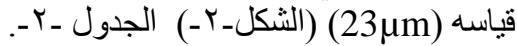

كما تبين من النتائج ان طفيلي Hepatozoon canis كان بشكل شبه دائري الى بيوضي داخل العدلات اذا كان قياسه ضمن المدى

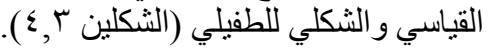

كما سجلت بعض الحالات التي ظهر فيها اصابة مزدوجة بكل من طفيلي Trypanosoma evansi وطفيلي Hepatozoon canis (الثكل 0) كما اظهرت النتائج وجود طفيلي Trypanosoma.evansi في المسحات المحضرة من طبقة الخلايا اللمفية (الثكل-7 -)

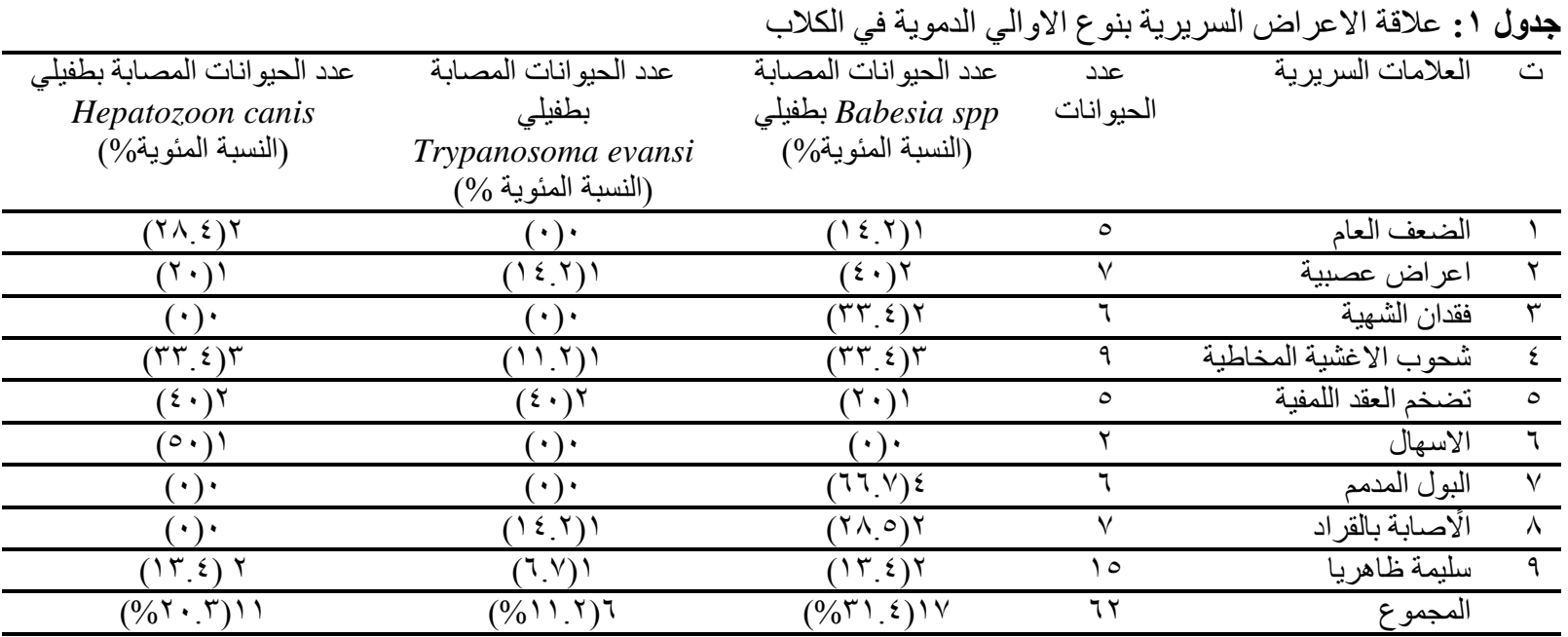

جدول Y : قياسات طفيلي Trypanosoma evansi في الكلاب

\begin{tabular}{lcccccc}
\hline Biometrical measurement & PK & KN & PN & NA & F & L \\
\hline Minimum & 1.8 & 2.4 & 4.3 & 5.1 & 8.1 & $21.7 \mu \mathrm{m}$ \\
\hline Maximum & 2.3 & 2.8 & 4.7 & 5.9 & 8.6 & $24.3 \mu \mathrm{m}$ \\
\hline Mean & 2.05 & 2.6 & 4.5 & 5.5 & 8.35 & $23 \mu \mathrm{m}$ \\
\hline
\end{tabular}

$\mathrm{PK}=$ distance from posterior end to kinetoplast

$\mathrm{KN}=$ distance from kinetoplast to middle of nucleus

$\mathrm{PN}=$ distance from posterior end to middle of nucleus

$\mathrm{NA}=$ distance from nucleus to anterior extremity

$\mathrm{F}=$ Free flagellum

$\mathrm{L}=$ total length including free flagellum 
و عند مقارنة نسبة الاصسابة بالفئات العمرية بينت النتائج ان اعلى نسب اصابة كانت في الحيوانات كبيرة العمر لكافة الاو الي الدموية

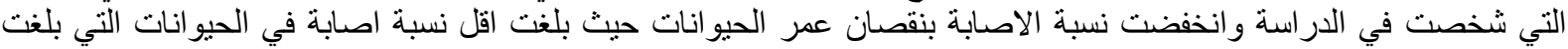
اعمار ها 7 اشهر - أسنة وبفروق معنوية واضحة بين الفئات العمرية الكبيرة والصغيرة.

جدول rا يبين علاقة نسبة الاصدابة بالاو الي الدموية بالفئات العمرية للكلاب

\begin{tabular}{|c|c|c|c|c|c|}
\hline $\begin{array}{c}\text { عدد الحيو انات المصابة } \\
\text { Hepatozoon canis } \\
\text { (النسبة المئوية \% }\end{array}$ & $\begin{array}{c}\text { عدد الحيو انات المصابة } \\
\text { Trypanosoma evansi } \\
\text { (النسبة المئوية \% } 1 \text { (ابلية }\end{array}$ & $\begin{array}{c}\text { عدد الحيو انات المصابة } \\
\text { Babesia spp } \\
\text { Baيلية النسبية المئوية\%) }\end{array}$ & الحيو انا & الفئات العمرية & $ت$ \\
\hline${ }^{a}(1 \cdot .0)^{r}$ & $\mathrm{a}(\cdot) \cdot$ & ${ }^{a}(Y) \leqslant$ & 19 & 7 اشهر - اسنة & 1 \\
\hline $\mathrm{a}(Y \nearrow, Y) \varepsilon$ & $\mathrm{a}(\nearrow, \mathrm{V}) \mathrm{l}$ & a(rT.s)o & 10 & اكبر من سنةـ سنة ونصف & r \\
\hline $\left.\mathrm{b}^{\mathrm{b}} \mathrm{Y}^{0}\right)^{0}$ & ${ }^{\mathrm{b}}(\Gamma \cdot)^{\mathrm{T}}$ & $\mathrm{b}_{(}(\varepsilon \cdot)^{\wedge}$ & $r$. & اكبر من سنة ونصف - r & $r$ \\
\hline$\left.(\% r \cdot r)^{\prime}\right) 1$ & $(\% \backslash 1 . r)^{\top}$ & $(\% \Gamma) . \varepsilon) \backslash \vee$ & $0 \leqslant$ & المجموع & \\
\hline
\end{tabular}

p

بينت نتائج علاقة نسبة الاصابة بالاو الي الطفيلية بجنس الحيوانات ان اعلى نسب اصابة سجلت بالذكور مقارنة بالاناث ولجميع

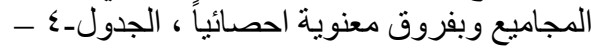

جدول ؟: علاقة نسبة الاصابة بالاو الي الدموية بجنس الكلاب

\begin{tabular}{|c|c|c|c|c|c|}
\hline $\begin{array}{c}\text { عدد الحيو انات المصابة } \\
\text { Hepatozoon canis } \\
\text { بطفيلية النسبة المئوية \% }\end{array}$ & $\begin{array}{c}\text { عدد الحيو انات المصنابة } \\
\text { Trypanosoma evansi } \\
\text { (النسبة المئوية \%) }\end{array}$ & $\begin{array}{c}\text { عدد الحيو انات المصابة } \\
\text { Babesia spp } \\
\text { (النسبة المئوية \% } 1 \text { (النيلية }\end{array}$ & الحيو انات & الجنس & $ت$ \\
\hline${ }^{a}(Y \circ . \vee)^{q}$ & ${ }^{a}(1 \leqslant . Y)^{0}$ & $\mathrm{a}(\{r . \wedge 0) 10$ & ro & ذكور & 1 \\
\hline $\mathrm{b}(1 \cdot .0)^{r}$ & $\mathrm{~b}(0, r) l$ & $\mathrm{~b}(1 . .0 r)^{r}$ & 19 & اناث & r \\
\hline$(\% r \cdot r) \backslash l$ & $\left(\% /{ }^{\prime}, r\right)^{\top}$ & $\left(\%{ }^{\top}, \varepsilon\right) \backslash \vee$ & $0 \leqslant$ & المجموع & \\
\hline
\end{tabular}

p p الاختلاف بالاحرف يعني ان القيم تختلف معنويا تحت مستوى احتمال

اما بالنسبة لعلاقة نسبة الاصابة بسلالة الكلاب التي خضعت للابلة استة فتبين ان نسبة الاصابة بمجمل انواع الاوالي الدموية كانت مرتفعة في سلالة الكلاب كبيرة الحجم ، في حين ان السلالات صغيرة الحجم كانة الت نسبة الاصدابة الكلية بالاو الي الاموية منخفضة ، جدول -

جدول ๑: علاقة نسبة الاصدابة بالاو الي الدموية بسلالة الكلاب

\begin{tabular}{|c|c|c|c|c|c|}
\hline $\begin{array}{c}\text { عدد الحيو انات المصابة } \\
\text { بطفية canis } \\
\text { Hepatozoon can النسبة المئوية \% }\end{array}$ & 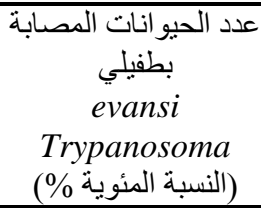 & 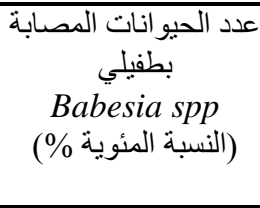 & 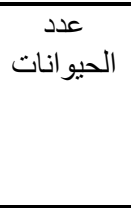 & السلالة & $ت$ \\
\hline$(10)^{r}$ & $(10)^{r}$ & $(\leqslant 0)^{9}$ & $r$. & $\begin{array}{c}\text { كلب الر اعي الالماني } \\
\text { German Shepherd }\end{array}$ & 1 \\
\hline$(r r . \varepsilon)^{\varepsilon}$ & $(\wedge . \varepsilon) !$ & $(17 . \vee)^{r}$ & TY & كلب المالينو البلجيكي & $r$ \\
\hline$(r \cdot)^{r}$ & $(1 \cdot)^{1}$ & $(\Gamma \cdot)^{\Gamma}$ & 1 . & كلب الهسكي السيبيري Husky & $r$ \\
\hline$(\mid \leq . r) !$ & $(\mid \leq . r) !$ & $(Y \wedge .0)^{Y}$ & $\mathrm{~V}$ & كلب رود ويلر Rod wheeler & $\varepsilon$ \\
\hline$(\cdot) \cdot$ & $(\cdot) \cdot$ & $(Y \cdot)^{\prime}$ & 0 & كلب تيرير Terrier & 0 \\
\hline$\left(\% r \cdot r^{r}\right)^{\prime \prime}$ & $(\% / 1 . r)^{\top}$ & $\left(\%{ }^{\top} . \varepsilon\right) \backslash \vee$ & $0 \xi$ & & \\
\hline
\end{tabular}




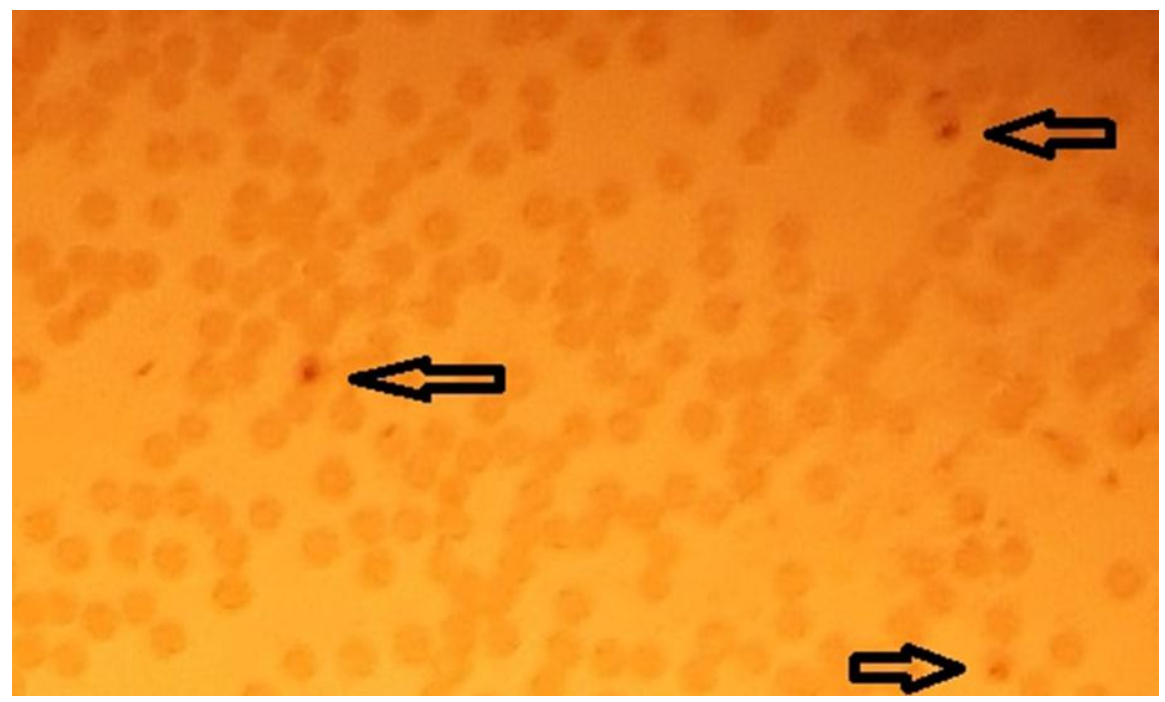

100X Babesia spp الثكل 1: يبين طفيلي

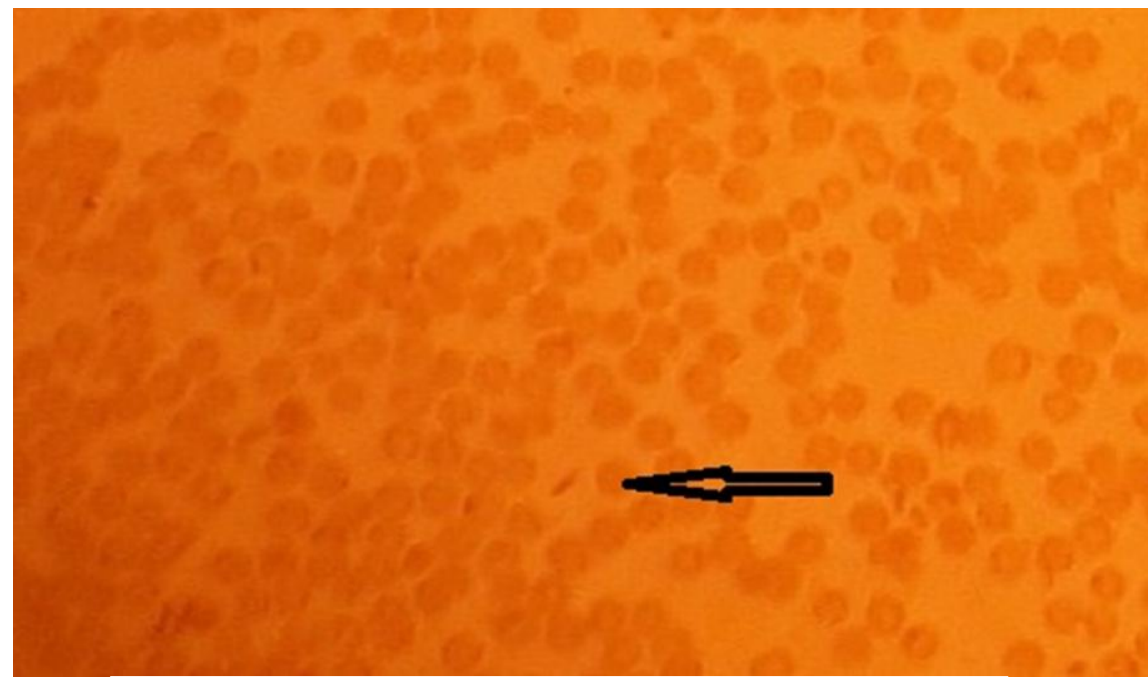

100X Trypanosoma evansi الثكل r : يوضح طفيلي

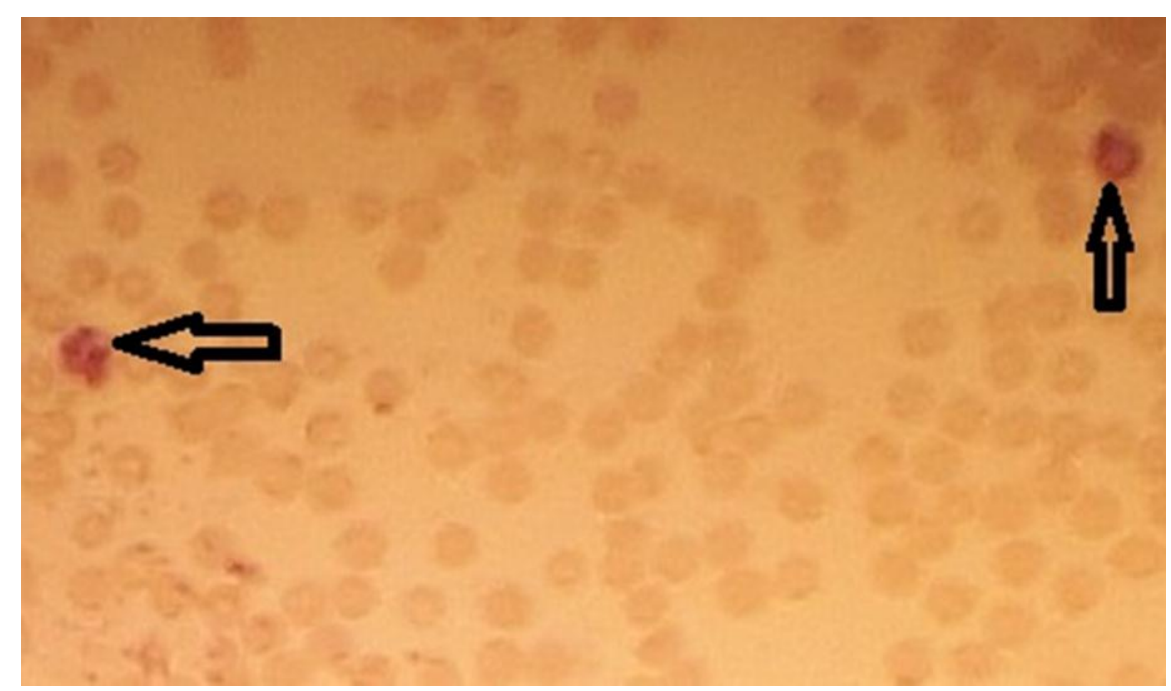

100X Hepatozoon canis الثكل r: ييين طفيلي 


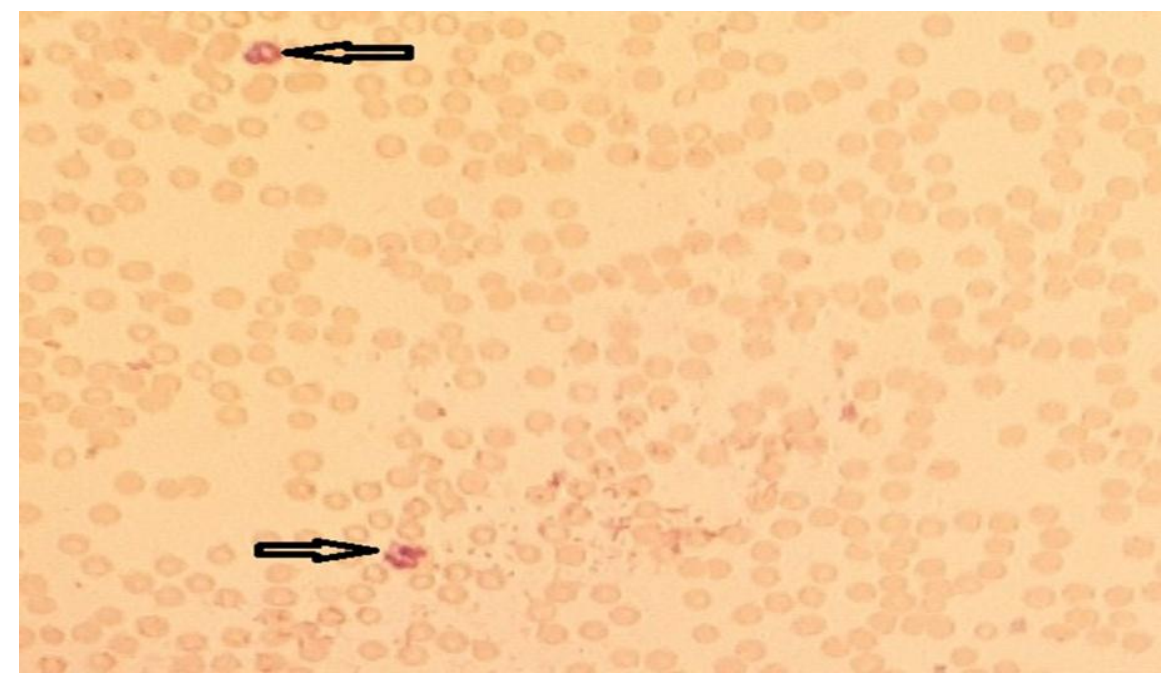

100X Hepatozoon canis الشكل ؛ : يبين طفيلي

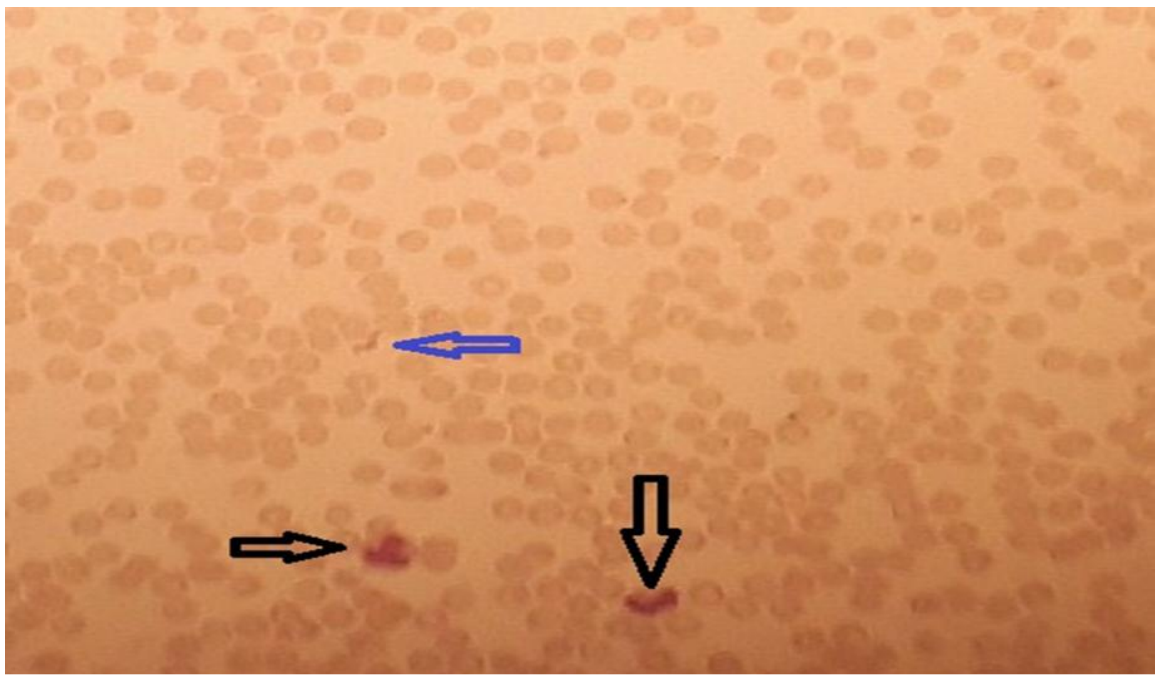

100X Trypanosoma evansi \& Hepatozoon canis الثكل •: يبين الاصابة المزدوجة بكل من طفيلي

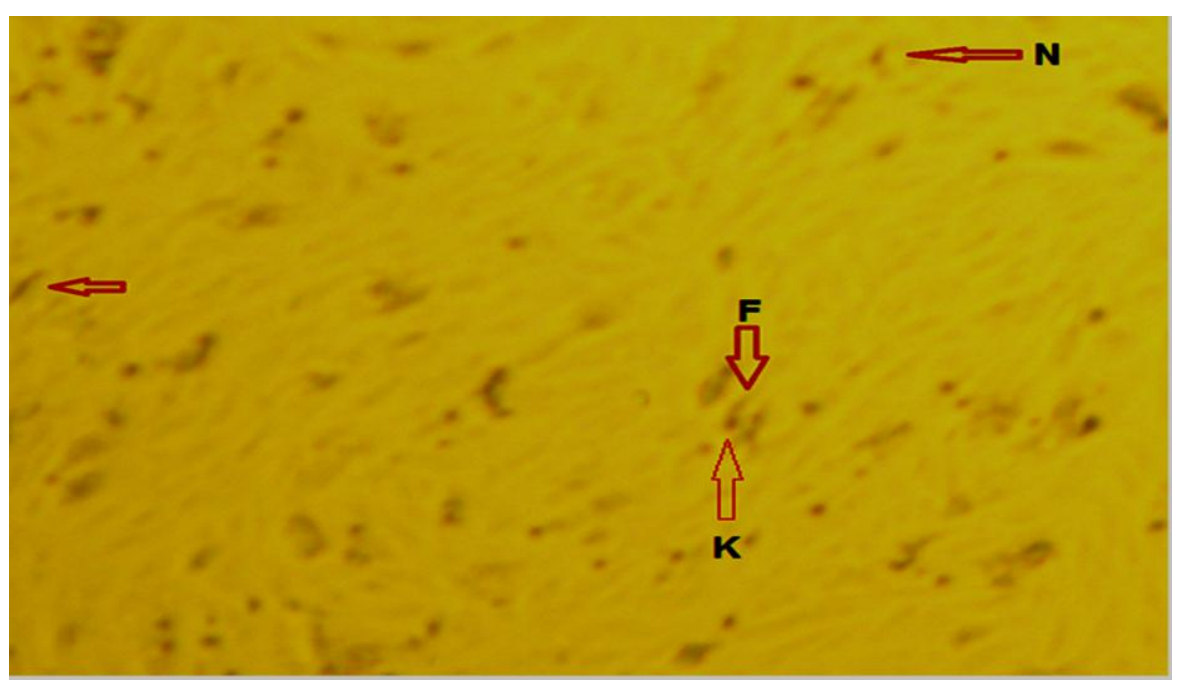

الشكل ؟: يبين طفيلي Trypanosoma evansi في مسحة تم تحضير ها من طبقة الخلايا اللمفية 100X الاحرف التالية تعني: N=Nucleus, K=Kinetoplas, F=Flagella 
الحيو انات المصابة بالطفيلي والتي لا نظهر الاعراض المرضية (حاملة للمرض)، اذ بينت نتائج دراستتا ارتفاعا بنسبة الاصابة

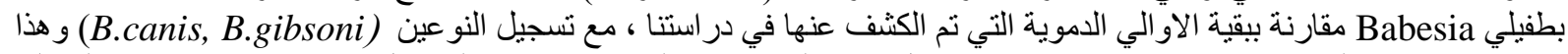

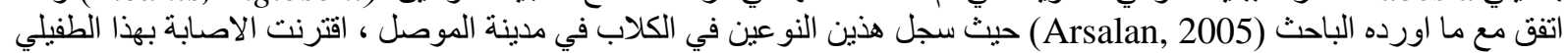

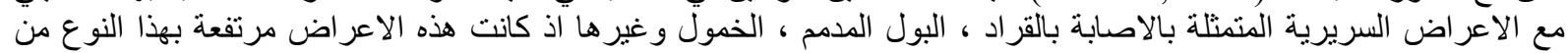

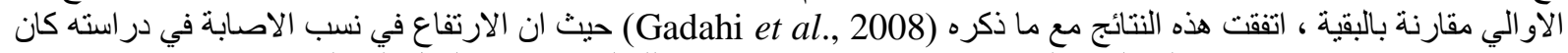

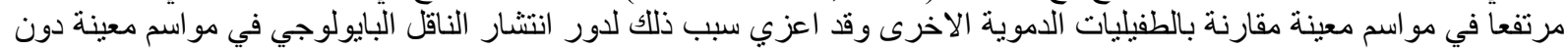

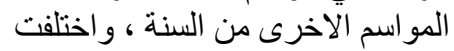

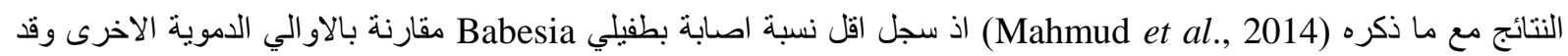
اعزي السبب الى الاختلاف في الانتشار الجغر افي بين بلدان العالم.

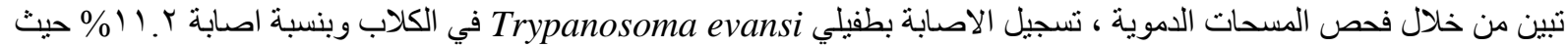

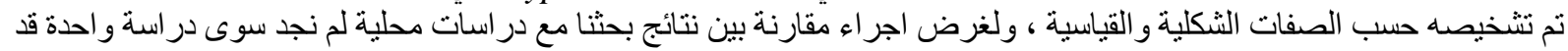

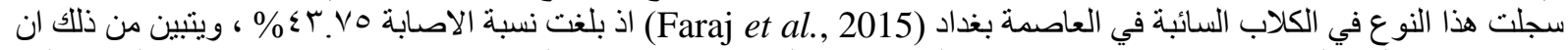

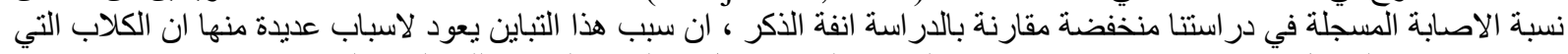

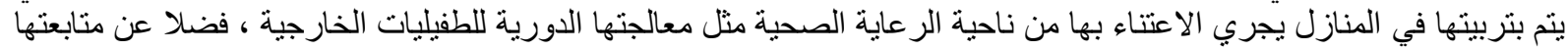

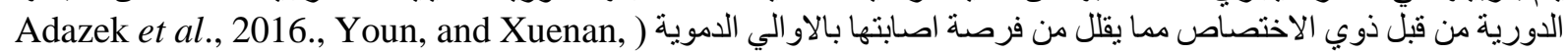

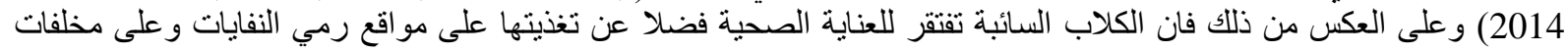
المجازر و التي تكون غنية بالنو اقل البايولوجية مما يزيد من نسب البـ الصابتها.

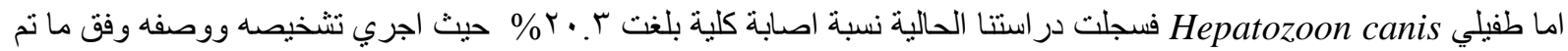

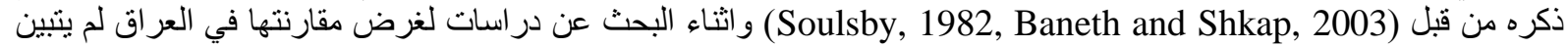
وجود اي در اسة مسبقة حول هذا الطفيلي ، الا ان هذا الطفيلي جرى تسجيله في الدول المجاورة للعراق مثل تركيا (B) (

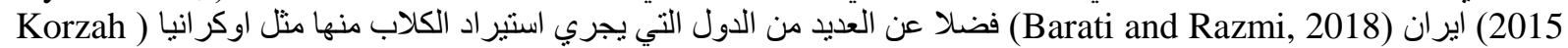

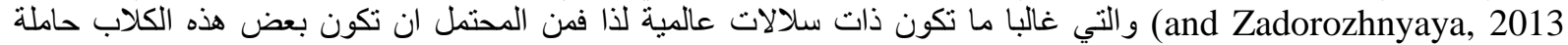
للاصـابة وتم ادخال الطفيلي من خلالها الى القطر لذا يعد من الامر اض الط الطارئة الى العراق.

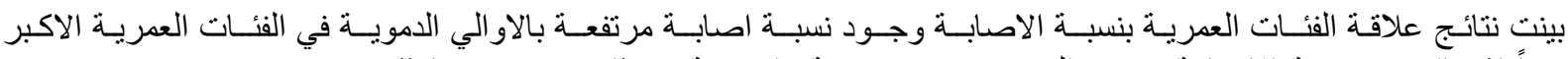

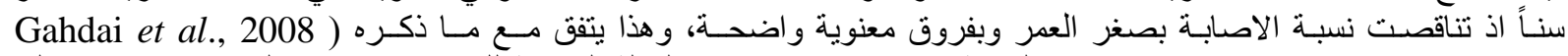
. Mahmud et al., 2014)

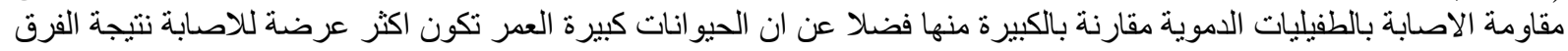

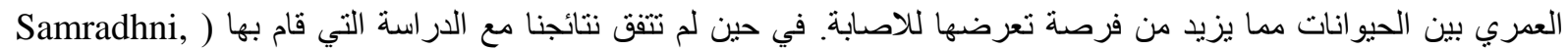

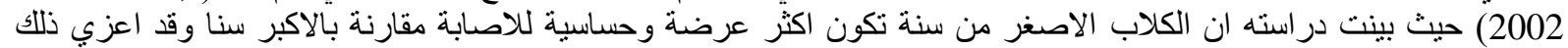

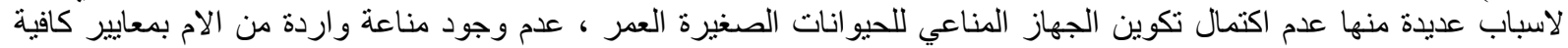

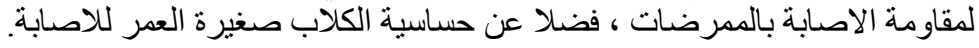

اظهرت نتائج علاقة نسبة الاصابة بالاو الي الدموية مع جنس الحيوانات حيث تبين ان نسبة الاصابة بالذكور اعلى مما هو عليه بالاناث

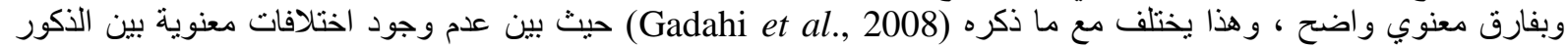

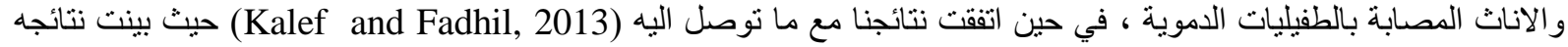

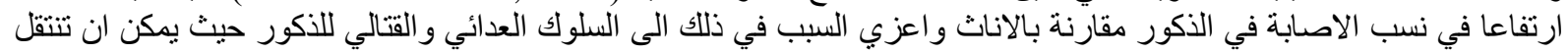

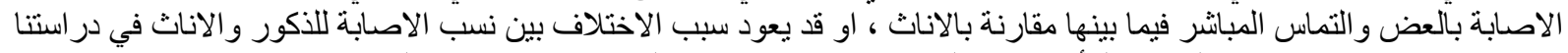

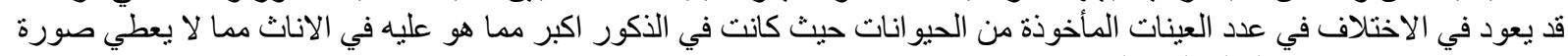
حقيقية عن نسبة الاصدابة الفعلية لكلا الجنسين.

تبين من خلال در اسة العلاقة ما بين نسبة الاصابة بالاو الي الدموية بسلالة الكلاب ان نسبة الاصابة كانت مرتفعة في السلالات كبيرة

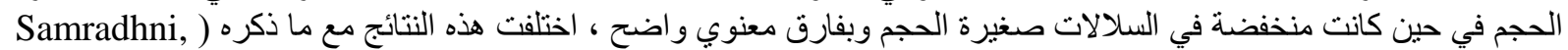

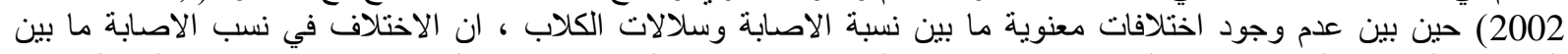

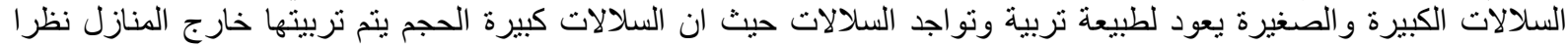

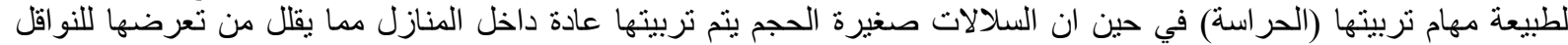
البايولوجية للاو الي الدموية وبالتالي تتخفض في نسبة الاصنية الصابة فيها (مشاهدات حقلية للباحث). 


\section{REFERENCES}

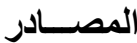

Adaszek, L.; Obara, G.; Piech, T. and Kalionwiski, M. (2016): Possible vertical transmission of Babesia canis canis from a bitch to her puppies case report. Veterinarian.medicinia, 61(5): 263266.

Arsalan, S.H. (2005): Clinical, haematological and biochemical studies of some blood protozoa in dogs in Ninveh. Iraqui. J. Vet. Sci, 19(1): 63-77.

Aydin, MF.; Sevinc, F. and Sevinc, M. (2015): Molecular detection and characterization of Hepatozoon spp. in dogs from the central part of Turkey. Ticks Tick Borne Dis. 6(3): 388-92.

Baneth, G. and Shkap, V. (2003): Monozonic cysts of Hepatozoon canis. J. Parasitol, 89(1): 3-9.

Barati, A. and Razmi, GR. (2018): A Parasitologic and Molecular Survey of Hepatozoon canis Infection In Stray Dogs In Northeastern Iran. J. parasitol. 104(4): 413-417.

Coles, E.H. (1986): Veterinary clinical pathology. $4^{\text {th }}$ ed. WB. Sanndar company, Philadelaphia

Duarte, S.C.; Parente, J.A.; Silivia, O.J.; Jayme, V.S. and Linhares, G.F. (2016): Molecular diagnosis of Hepatozoon canis in symptom- atic dogs in the city of Goiania. Goisa, Brazile.

Faraj, A.; Afkar, M. and Al-Amery, A.M. (2015): Prevalence trypanosomiasis of stray dog in Baghdad city, Iraq inter city, Iraq. International Journal of Recent Scientific. Research 6(11): 7206-7208.

Gadahi, A.A.; Arijo, G.; Abubakar, M.; Javaid, S.B. and Arshed, M.J. (2008): Prevalence of Blood parasites in stray and pet Dogs in Hyderabad Area: Comparative sensitivity different Diagnostic techniqes for the detection of microfilaria Veterinary World, Vol.1(8): 229-232.

Hoare, C.A. (1972): The Trypanosomiase of mammals. A zoological

Kalef, D.A. and Ali, I.F. (2013): Incidance of Babesia gibsoni in stray dogs (Iraq). OJVR,17(1):32-35.

Korzh, A.P. and Zadorozhnyaya, V.Y. (2013): The biological characterstics of representatives of the genus Hepatozoon (Apicomplexa), Adeleorina in the marsh frog separate populations of Zaporizhzhya region. Vestnik zoologii, 47(2): 34-39.

Lan, H.; Xiaoyan, M.; Jinfang, H.; Yuan, H.; Pei, H.J.; Junwei, H.; Long, Y.; Ngabu, M.; Ligang, S. and Junlong, Z. (2017): First Molecular Detection of Babesia gibsoni in Dogs from Wuhan, China. Front Microbiol. 8: 1577.

Mahmud, M.A.A.; Belal, S.M. and Uddin, F.M.J. (2014): Prevalence of protozoan disease in pet dogs at district veterinary hospital, Siranj, Bangaldish. Bangl. J. Vet. Med. 12 (2): 191-196.

MICHAL, F.; RAFAL, S. and WŁODZIMZ, K. (2010): Analysis haematological Abnormalities observe in dogs infected by a large Babesia Bull Vet Inst Pulawy 54, 167-170.

Saleem, A.H. (2007): A review of confirmed pathogens of dogs and cats Iraq. Bas, J., Vet., Res, 6,(2):156-162.

Samradhni, D.D. (2002): Studies on haemoprotistant infection in canines at Nagpur (Maharashtra). Master thesis, University of Nagpur India.

Soulsby, E.J.L. (1982): Helminths, Arthropods, and Protozoa, of domesticated animals, $7^{\text {th }}$ edn, ElBS and Bailliere Tindall And Casael Ltd. London.

Youn, K. and Xuenan, X. (2014): New Molecules in Babesia gibsoni and Their Application. for Diagnosis, Vaccine Development, and Drug Discovery. Korean J Parasitol. 52(4): 345-353. 